\title{
MRI findings and physical performance as predictors of flight-induced musculoskeletal pain incidence among fighter pilots
}

\author{
Harri Rintala ${ }^{1}$, Roope Sovelius ${ }^{2}$, Pirjo Rintala ${ }^{3}$, Heini Huhtala ${ }^{4}$, Simo Siitonen $^{5}$, Heikki Kyröläinen ${ }^{1,6}$ \\ ${ }^{1}$ University of Jyväskylä, Faculty of Sport and Health Sciences, Jyväskylä, Finland; ${ }^{2}$ Centre of Military Medicine, Satakunta \\ Air Command Health Clinic, Pirkkala, Finland; ${ }^{3}$ Central Hospital of Central Finland, Department of Physical Medicine and \\ Rehabilitation, Jyväskylä, Finland; ${ }^{4}$ School of Public Health, University of Tampere; Research Unit, Tampere University \\ Hospital, Tampere, Finland; ${ }^{5}$ Defence Staff, Medical Branch, Helsinki, Finland; ${ }^{6}$ National Defence University, Helsinki, \\ Finland
}

\section{Summary}

Study aim: The aim of this study was to evaluate the possible association of pre-career magnetic resonance imaging (MRI) findings and physical performance level with possible musculoskeletal disorders during jet flight training.

Material and methods: The study group consisted of 73 fighter pilots who had undergone pre-career cervical and lumbar spine MRI. Physical performance of a subgroup of the pilots $(n=67)$ was measured initially at the same time and followed up to the fast jet training phase (ranging from 3.8 to 7.0 years). Musculoskeletal pain history during pilot training was taken from the medical charts. MRI findings and physical performance were associated with perceived clinical complaints during the follow-up.

Results: $82 \%$ of the cervical and $92 \%$ of the lumbar spines showed abnormalities at at least one disk level. MRI did not reveal significant cervical degeneration. Thirteen disk bulges in the lumbar spine were discovered, while 5 pilots had listhesis and/or osteophyte formation on the spine (lumbar vertebra 4/sacroiliac joint level, L4-SI). 41\% of the studied pilots suffered spinal symptoms during the follow-up, but only $16 \%$ and $17 \%$ of the cervical and lumbar MRI findings, respectively, were associated with subsequent symptoms. Endurance and strength levels were not, but lower body motor skills were, strongly (relative risk, RR 0.46) associated with a decreased number of flight-induced medical appointments in the early flight career.

Conclusions: Minor MRI findings have no predictable value in the very early flight career. Nevertheless, versatile, skills/ power-oriented exercises before the flight career seem to be occupationally beneficial in reducing musculoskeletal disorders.

Keywords: MRI - Disk degeneration - Cervical spine - Lumbar spine - Military pilot - Fitness

\section{Introduction}

Pilots of modern fighters encounter high $+\mathrm{Gz}$ forces. The occupational work load may exceed the limits of muscular performance, thus causing musculoskeletal pain and/ or disability in the daily service. Combat flying places high demands on both the pilots' spinal structure and physical performance. Practical methods attempting to predict possible flight-induced musculoskeletal problems later during the flight career include spinal column magnetic resonance imaging (MRI) and specific, occupationally oriented physical fitness testing during the pilot selection process.

Since a number of reports indicate that $\mathrm{Gz}$ exposure has caused acute in-flight pain $[15,27]$ and injuries $[16,21]$ as well as premature degeneration $[13,24]$, the health of spinal structures should be assessed during selection of pilots. The NATO Research and Technology Organization has recommended that some form of spinal diagnostic imaging should be included in the evaluation of recruits in order to detect any spinal abnormalities [8].

Findings with imaging techniques are common among asymptomatic young people, but there are contradictory reports among pilots. Among a selected population of 232 Norwegian military pilot recruits, the spinal radiographs of only $1 / 3$ of them were considered normal, while $1 / 10$ of recruits were rejected from flight training due to radiological findings [2]. A German report covering 10922 asymptomatic pilot recruits stated that no more than $3 \%$ of them were "normal"; i.e., they had no morphological changes in spinal X-rays [11]. In a group of young inexperienced Swedish pilots (average age 23 years, 220 flight 
hours), cervical MRI showed a low frequency of occurrence of low-grade degenerative lesions [23]. A Finnish study showed that there were no significant changes in pilots' spinal column during 13 years of follow-up, but only 12 pilots were followed [28].

Magnetic resonance scans are more accurate than conventional radiographs due to their ability to demonstrate also intervertebral disk pathology. Other imaging techniques such as radiography, myelography and computed tomography (CT) may provide complementary information in selected cases [18]. A mild degree of degenerative changes is paraphysiological and should be considered pathological only if abnormalities determine symptoms [10]. The significance of radiological findings needs to be further discussed. Recent literature has suggested that nerve compression is an important finding that may contribute to the prediction of symptoms [9]. An association between recent lower back pain and endplate changes, disk degeneration, annular tears or facet degeneration was not found to be a risk factor in a three-year follow-up. In addition, only limited information of the associations between occupational musculoskeletal disorders, induced disability and physical performance is available among military pilots. However, it has been suggested that a high level of muscular fitness protects against disability but not work-related disorders [25].

There is only limited epidemiological evidence of the associations between pilots' physical performance and occupational musculoskeletal disorders [25]. Long-term, comprehensive trials and/or follow-ups on this issue do not exist in the modern aeromedical literature, so there is an evident requirement for this kind of study design. Therefore, the aim of the present study was to grade and examine pre-career MRI findings among pilot candidates and evaluate their importance in the prediction of workrelated musculoskeletal disorders during subsequent flight training. Simultaneously, the baseline level in pilots' occupational physical performance and its association with perceived flight-induced symptoms during the early flight career were determined.

\section{Material and methods}

Data were obtained from 73 out of 88 pilots who had undergone pre-career MRI of the cervical and lumbar spine. Their mean age during the flight phase was $26.9 \pm 1.5$ years, height $178 \pm 5 \mathrm{~cm}$, body mass $77.0 \pm 7.0 \mathrm{~kg}$, and body mass index (BMI) $24.1 \pm 1.8 \mathrm{~kg}$ $\mathrm{m}^{-2}$. Their total mean accumulated flying time was 673 $\pm 228 \mathrm{~h}$ and time in high-Gz capable aircraft (Hawk and F-18) was $504 \pm 120 \mathrm{~h}$. Fifteen pilots were excluded from the analysis. Two of them had ejected during their career, 3 pilots lacked cervical or lumbar MRI or a complete medical history, and 10 of them had been pulled out of fighter training due to various reasons. The excluded pilots did not differ from the study sample in their age or BMI. Two pilots, whose fighter pilot training had been terminated due to back problems, were, however, included in the analysis.

A retrospective, evidence-based analysis of neck and back pain history during early fighter pilot training was undertaken using information from medical charts (pilots' medical appointments for flight-induced pain) [25]. The average follow-up time after the MRI examination was 6.5 years (range from 3.8 to 7.0 years). The MR images were analysed by one MRI-experienced medical doctor, specialized in physical rehabilitation, in a blinded fashion independently from clinical information. The MR examinations were performed with a $1.0 \mathrm{~T}$ device (GE Signa Horizon, UK), and they included the T1 and T2 weighted sagittal and axial MRI of the cervical and lumbar spine. The grading of findings in the cervical spine [17] is presented in Table 1, and the grading of the lumbar spine findings [29] is presented in Table 2. The design of this study was approved by the Ethical Committee of the Central Finland Health Care District (Jyväskylä, Finland) before data collection.

The MRI findings were linked with clinical complaints during the follow-up. These complaints were graded from 0 to $2(0=$ no symptoms, $1=$ moderate and temporary symptoms, and $2=$ severe or continuous disorders, e.g. sciatic symptoms, confirmed disk bulges with clinical symptoms). An experimental physical performance test protocol was conducted in the pilot selection process for assessing the flight cadets' "occupational specific fitness" characteristics. All tests were conducted by an aerospace physiologist in the Aeromedical Centre (Helsinki, Finland). Sixty-seven pilots, who already had MRI results available within 6 months of the tests, were followed up on average for 5.4 years (ranging from 3.8 to 7.0 years). The drop-out of six pilots was due to the lack of some physical performance tests. The tests consisted of

Table 1. Grading of MRI findings at the cervical spine level

\begin{tabular}{lcc}
\hline Disk nucleus signal & Prolapse & Bone marrow signal \\
\hline $1=$ no signal loss & $1=$ no prolapse & $1=$ no intensity change \\
$2=$ moderate signal loss & $2=$ protrusion & $2=$ intensity change \\
$3=$ total loss & $3=$ prolapse & \\
\hline
\end{tabular}


Table 2. Grading of MRI findings at the lumbar spine level

\begin{tabular}{lc}
\hline Anterior column & Posterior column \\
\hline $1=$ normal & $1=$ no facet joint degeneration \\
$2=$ disk nucleus signal loss & $2=$ facet joint degeneration without stenosis \\
$3=$ protrusion & $3=$ facet joint degeneration with stenosis or spondylolysis \\
$4=$ prolapse & \\
$5=$ degenerative listhesis & \\
6 & $=$ osteophytes
\end{tabular}

evaluation of aerobic power by indirect maximal oxygen uptake with a bicycle ergometer (Ergoline 800, Bitz, Germany) [26], anaerobic power by counter movement jump on a contact mat with additional motor control evaluation, isometric maximal neck and trunk force measurements [3], and $1 \mathrm{~kg}$ medicine ball throw through infra-red photo cells with a maximal effort (Newtest Trunk/Neck force and Powertimer, Kempele, Finland) [22]. The medical appointments due to the flight-induced pain were associated with the pilot candidates' physical performance level during the selection phase.

The level of agreement (kappa value) in the radiological rating of every MR image was calculated by the same reader (physiatrist) in two separate readings. The intraobserver agreement of MRI ratings was $92 \%$ (0.58). Binary logistic regression analysis was used to determine the association of MRI findings with the incidence of symptoms. The odds ratios (OR) and 95\% confidence intervals are presented. An evidence-based flight-induced pain incidence curve was created according to the real medical appointments, and a Poisson univariate regression model was used to predict (RR, relative risk; 95\% CI) the flightrelated pain incidence with the level of physical performance. The significance level was set at $p \leq 0.05$ in all statistical analyses.

\section{Results}

Eighty-two percent of the cervical and $92 \%$ of the lumbar spines were observed to have abnormalities at at least one disk level. No significant cervical degeneration was found in the pre-career MRIs. All rated changes in the cervical spine were indicated by only moderate signal loss in intervertebral disks, and there were no disk protrusions or bone marrow signal intensity changes. These findings were most common at the cervical vertebra 5-thorax vertebra 1 (C5-Th1) level. Rated degenerative changes in the lumbar spine were found as follows: 41 subjects had a protrusion at at least one disk level ( $56 \%$ of the subjects), 2 subjects

Table 3. Detailed relative occurrence of MRI changes [\%] at each cervical (C) and lumbar (L) spine level before flight service. The same subject can be included in multiple categories $(\mathrm{n}=73)$

\begin{tabular}{lccccccc}
\hline & C1-2 & C2-3 & C3-4 & C4-5 & C5-6 & C6-7 & C7-T1 \\
\hline No changes & 99 & 97 & 97 & 88 & 66 & 42 & 40 \\
Signal loss in a disk & 1 & 3 & 3 & 12 & 34 & 58 & 60 \\
Prolapse & 0 & 0 & 0 & 0 & 0 & 0 & 0 \\
Bone marrow signal intensity change & 0 & 0 & 0 & 0 & 0 & 0 & 0 \\
\hline & L1-2 & L2-3 & L3-4 & L4-5 & L5-S1 & & \\
\hline No changes & 100 & 100 & 95 & 51 & 27 & & \\
Signal loss in a disk & 0 & 0 & 5 & 34 & 25 & & \\
Protrusion & 0 & 0 & 0 & 14 & 42 & & \\
Prolapse & 0 & 0 & 0 & 1 & 1 & & \\
Listhesis & 0 & 0 & 0 & 0 & 4 & & \\
Osteophytes & 0 & 0 & 0 & 0 & 0 & & \\
Facet degeneration with stenosis* & 0 & 0 & 0 & 0 & 3 & & \\
\hline
\end{tabular}

*The graded facet degenerations were related to subjects with listhesis, and the figure of listhesis includes the subjects with facet degeneration. 
had a prolapsed disk ( $2 \%)$, and 3 subjects had a listhesis at the L5-SI-level (4\%).

One third of the pilots had a medical appointment due to flight-induced pain during the first 5.4 years from the beginning of the service, but only $16.5 \%$ (OR 3.8, CI95\%: 0.8 to 18.7 ) of the cervical and $16.4 \%$ (OR 2.8, CI95\%: 0.7 to 11.0) of the lumbar MRI findings were associated with subsequent symptoms (Fig. 1). Higher-degree MRI findings were associated with subsequent severe symptoms only in $6.8 \%$ (OR 1.7, CI95\%: 0.4 to 7.1 ) of the pilots. Most of them with more significant findings, e.g., at least protrusion (grade 3 or more), were asymptomatic during the follow-up (28 out of 37 ).

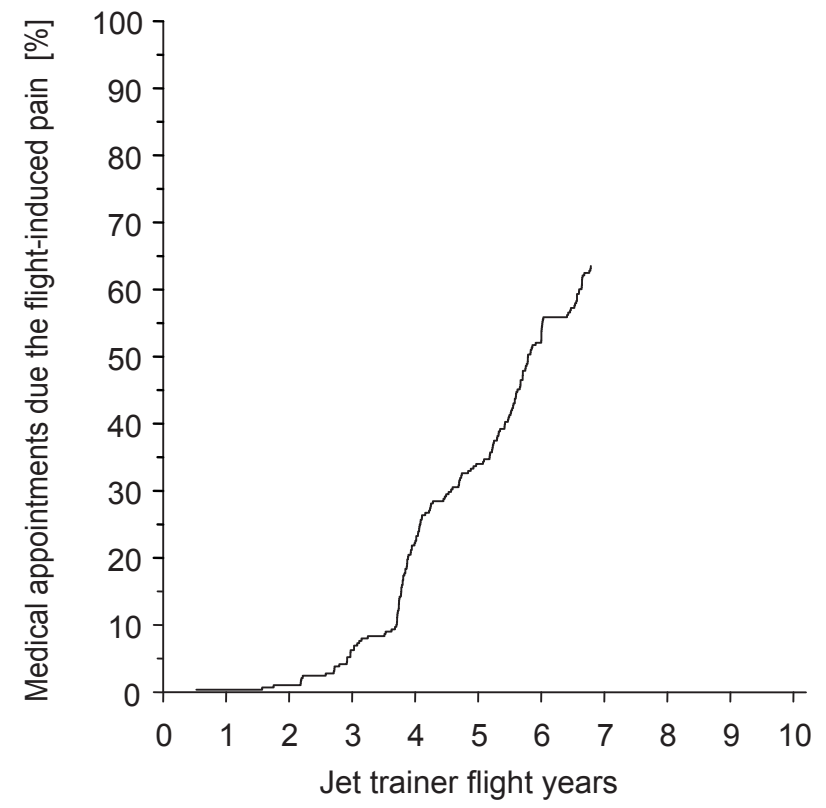

Fig. 1. Association between jet trainer flight years and medical appointments because of flight-induced, flight service disturbing musculoskeletal pain $(\mathrm{n}=67)$

Out of 2 pilots who showed severe or continuous cervical symptoms (grade 2), the first one had a normal cervical MRI, and the other one had signal loss in the three lowest cervical intervertebral disks. There were 8 pilots with clinical outcomes graded as 2 . Their lumbar MRI findings included 2 normal, 1 signal loss, 4 protrusions at at least one disk level, and one listhesis. Normal MRI was associated with asymptomatic follow-up in $92 \%$ of cases in the cervical spine and in $60 \%$ of cases in the lumbar spine. Furthermore, no significant association was noted between pre-flight career MRI findings and flight-induced medical appointments in the early flight syllabus.

Table 4 shows the pilots' main characteristics in physical performance before the follow-up. In Table 5, the associations between different physical performance test results and the incidence of any flight-induced medical appointment are presented. The pilots' fluent motor control in the anaerobic jumping test and neck flexion forces had the strongest associations with flight-induced medical appointments and thus in the restriction of the occupational related musculoskeletal disorders.

Table 4. Fighter pilots' $(n=67)$ physical performance and background characteristics during the selection phase

\begin{tabular}{lll}
\hline Variable & Mean & SD \\
\hline Age, years & 18 & - \\
BMI & 24.1 & 2.3 \\
Aerobic power [W/kg] & 4.2 & 0.5 \\
Anaerobic power [W/kg] & 24.9 & 7.2 \\
Neck flexion [N] & 221 & 52 \\
Neck extension [N] & 294 & 48 \\
Trunk flexion [N] & 660 & 156 \\
Trunk extension $[\mathrm{N}]$ & 900 & 146 \\
Ball throw time [ms] & 220 & 15 \\
\hline
\end{tabular}

Table 5. Association (relative risk, RR) between the pilots' different physical performance test results and their medical appointments because of any flight-induced musculoskeletal disorder during the follow-up $(n=67)$

\begin{tabular}{lll}
\hline Test & $\mathrm{RR}$ & $\mathrm{p}$ \\
\hline Aerobic power & 1.0 & 0.009 \\
Anaerobic power & 1.01 & 0.393 \\
*motor control in jumping & 0.46 & $<0.001$ \\
Neck flexion & 0.96 & $<0.001$ \\
Neck extension & 1.02 & 0.084 \\
Trunk flexion & 0.99 & 0.106 \\
Trunk extension & 1.00 & 0.595 \\
Ball throw time & 1.00 & 0.247 \\
\hline
\end{tabular}

\section{Discussion}

This study lays the basis for a flight-career long follow-up by determining the essential information of the two practical occupational variables for future purposes.

Neither the MRI findings nor the occupational specific physical performance indisputably predicted flightinduced musculoskeletal pain at a statistically significant level during the early years of the flight service. Even most of the pilots with higher-degree MRI findings in the lumbar spine were asymptomatic during the follow-up despite having undertaken high-Gz flying. So far in our study, Gz exposure seems to have no bearing on musculoskeletal symptoms irrespective of the occurrence of morphological changes in the spinal structure. These results are similar to 
those of earlier non-pilot studies $[5,6,9,14]$ and some observations made among Gz-exposed populations [7, 24]. However, normal cervical MRI seems less likely to be subsequently associated with neck pain.

The incidence of cervical disk degeneration changes was higher in this study compared with earlier similar studies, but the degree of degenerative changes was lower $[5,19,28]$. This may be due to the fact that the subjects represented a population selected with very special criteria. Most changes were located in the lower cervical spine, which is contrary to the study of Abdulkarim et al. [1] which suggested that findings in the cervical spine are distributed evenly at a young age but tend to occur in the lower disks with increasing age. If imaging of the neck and back were used in selection, some challenging questions - such as what would be considered critical or predictive findings, and how criteria for the rejection of asymptomatic recruits with pathological findings should be established - would need to be thoroughly discussed.

The lumbar spine MRI findings and their outcomes were also well in line with earlier studies [6,9]. The level of changes seems to be concentrated in the lower lumbar spine. The incidence of musculoskeletal symptoms either in the cervical or lumbar spine was obviously slightly lower than rates reported in the literature $[12,15]$. There might exist non-disabling symptoms that do not lead an individual to contact occupational health care services in the early phases of the flight syllabus. This might partially explain why the incidence of flight-induced medical appointments showed a steeply ascending trend in the present follow-up (Fig. 1). Secondly, this trend reflects quickly cumulating physical load in the later flight service, and thus may lead to an increasing amount of work-related, more disabling symptoms.

The follow-up time was not long enough in view of the cumulative individual $\mathrm{G}$ exposure. This is an interesting phenomenon in aerospace physiology and surely needs more research to be defined and measured. However, as clinical information was based on the pilots' medical records, there was no recall bias that may be a factor in any retrospective questionnaire.

Other occupational (non-Gz related) or leisure time physical loadings may also cause musculoskeletal symptoms and degeneration. Determinants of these types of phenomena are not adjustable during a pilot's career, and they have been reported to have only a modest influence on degenerative changes $[4,30]$. Symptoms other than flightinduced ones also affect pilots' fitness to fly and their quality of life. Although the effects of physical activity were not evaluated in this study, it is suggested that the correlation of physical activity with flight-related musculoskeletal disorders should also be examined in future studies.

The young pilots performed quite well in the physical performance tests compared to the literature $[3,26,28]$, but the average results were only barely satisfactory compared to typical endurance or strength sports athletic levels [20]. The physical performance, like the MRI findings, was mainly not associated with perceived pain, which reflects well the multifactorial nature of this issue. The absolute physical performance, compared to athletes, was poor or only satisfactory in different tests [22], but the pilots were quite good in terms of military performance, in general. Jumping skills seemed, however, to considerably reduce the number of flight-induced medical appointments. Obviously, overall exercising has created such a good body balance, core muscle stability and posture that it has proven to be occupationally beneficial. High neck flexion and extensor muscles' strength, as a protective factor, probably represent an ideal agonist-antagonist relationship; however, muscular balance was not measured in this study.

Excellent physical performance will reduce the induced work-related disability during the whole flight career [25]. However, due to the short-term design used in this study, it is so far impossible to conclude whether the measured physical performance and, especially, its changes are associated with perceived pain in the future. The pilots were obviously young, at the very beginning of their flight career, and no relevant controls existed.

Are there any predictable issues that could be regarded as warning signs of later spinal problems? Minor or even moderate changes may or may not result in symptoms during the later phases of a pilot's career. Studies among non-pilots indicate that nerve root contacts are the most predictable findings for pain. However, MRI findings are not predictive of the development or duration of back pain and vice versa. Individuals suffering from prolonged back pain may not have the greatest degree of anatomical abnormality [6]. Longer follow-up could yield more information about developing degenerative changes and their relationships with different phases of flight training (i.e. different G-forces). It could also be useful to assess the surrounding soft tissues such as muscle and fat mass and its location, and elastic structure including connective tissue types, to seek new associations between work-related symptoms and performance.

Some limitations must be discussed. The follow-up time was not equal for each subject, which resulted in deviation in completed training (flight hours) and Gz exposure. However, the pilots had completed their minimum basic and tactical jet training. Those ones whose career diverted from active flying with high performance aircraft or who had been pulled out from air combat training were excluded from the material. Thus, it was considered that the follow-up data were homogeneous enough to enable reliable evaluation of the outcome of the MRI findings and prediction of developing symptoms. 1.0 T MRI devices are also already substituted by modern equipment. Thus, it might be possible that the baseline findings might have 
been more accurate with the newest methods. Having only one reader to interpret images has its pros and cons. On one hand, there is no variation in opinions when a single reader performs grading; on the other hand, the absence of control readers may result in unproven opinions. Hence, it was considered that the use of just one reader in this study was a relevant method in terms of occupational medicine. Fitness tests were not conducted exactly at the same time as MR imaging, but the protocol was arranged during the same pre-flight phase with MRIs in the flight syllabus, in the same fitness test laboratory, and by the same test personnel and weekly calibrated equipment.

This may be one of the first studies where military pilots' MRI and physical performance were evaluated together in detail with later clinical outcome. As the results of this study do not indicate a clear correlation with the cervical or lumbar MRI, physical performance and subsequent symptoms in the early flight syllabus, more follow-up time for the syllabus is needed, especially up to active flight service and retirement years. Minor degenerative changes do not seem to cause severe disorders despite exposure to high Gz loadings, which suggests that it could be valuable to emphasize nerve root contacts and clearly deformed spinal structures in the pilot selection process. Clinical correlation is essential to determine the importance of abnormalities on magnetic resonance images, which should also be used to determine the surrounding tissues status, i.e. body fat and active tissue balance and the circumference of the active muscular tissue, not just to make notes on bone structure anomaly. Occupational-oriented physical performance and especially its changes should be included even in the basic military fitness testing or aeromedical examination procedures to clarify the importance in the fighter pilots' careerlong occupational performance.

\section{Conflict of interest: Authors state no conflict of interest.}

\section{References}

1. Abdulkarim J.A., Dhingsa R., Finlay D.B. (2003) Magnetic resonance imaging of the cervical spine: frequency of degenerative changes in the intervertebral disc with relation to age. Clin. Radiol., 58: 980-984.

2. Andersen H.T., Wagstaff A.S., Sverdrup H.U. (1991) Spinal X-ray screening of high performance fighter pilots. Aviat. Space Environ. Med., 62: 1171-1173.

3. Backman J., Häkkinen K., Ylinen J., Häkkinen A., Kyröläinen H. (2005) Neuromuscular performance characteristics of open-wheel and rally drivers. J. Strength Cond. Res., 19: 777-784.

4. Battie M.C., Videman T., Gibbons L.E., Fisher L.D., Manninen H., Gill K. (1995) 1995 Volvo Award in clinical sciences. Determinants of lumbar disc degeneration. A study relating lifetime exposures and magnetic resonance imaging findings in identical twins. Spine, 20: 2601-2612.

5. Boden S.D., McCowin P.R., Davis D.O., Dina T.S., Mark A.S., Wiesel S. (1990) Abnormal magnetic-resonance scans of the cervical spine in asymptomatic subjects. A prospective investigation. J. Bone. Joint. Surg. Am., 72: 1178-1184.

6. Borenstein D.G., O’Mara J.W. Jr., Boden S.D., Lauerman W.C., Jacobson A. Platenberg C., Schellinger D., Wiesel S.W. (2001) The value of magnetic resonance imaging of the lumbar spine to predict low-back pain in asymptomatic subjects : a seven-year follow-up study. J. Bone. Joint. Surg. Am., 83-A: 1306-1311.

7. Burns J.W., Loecker T.H., Fischer J.R. Jr., Bauer D.H. (1996) Prevalence and significance of spinal disc abnormalities in an asymptomatic acceleration subject panel. Aviat. Space Environ. Med., 67: 849-853.

8. Burton R. Cervical spinal injury from repeated exposures to sustained acceleration. Neuilly-sur-Seine. Cedex. France: NATO RTO-TR-4; 1999. AC/323(HFM)TP/9.

9. Elfering A., Semmer N., Birkhofer D., Zanetti M., Hodler J., Boos N., (2002) Risk factors for lumbar disc degeneration: a 5-year prospective MRI study in asymptomatic individuals. Spine, 27: 125-134.

10. Gallucci M., Limbucci N., Paonessa A., Splendiani A., (2007) Degenerative disease of the spine. Neuroimaging Clin. N. Am., 17: 87-103.

11. Hald H.J., Danz B., Schwab R., Burmeister K., Bahren W. (1995) Rontgenologisch nachweisbare Wirbelsaulenveranderungen asymptomatischer junger Manner [Radiographically demonstrable spinal changes in asymptomatic young men]. Rofo: Fortschr. Geb. Rontgenstr. Nuklearmed., 163: 4-8.

12. Hämäläinen O. (1999) Thoracolumbar pain among fighter pilots. Mil. Med., 164: 595-596.

13. Hämäläinen O., Vanharanta H., Kuusela T. (1993) Degeneration of cervical intervertebral disks in fighter pilots frequently exposed to high $+\mathrm{Gz}$ forces. Aviat. Space Environ. Med., 64: 692-696.

14. Jarvik J.G., Hollingworth W., Heagerty P.J., Haynor D.R., Boyko E.J., Deyo R.A. (2005) Three-year incidence of low back pain in an initially asymptomatic cohort: clinical and imaging risk factors. Spine, 30: 1541-1548; discussion 1549.

15. Kikukawa A., Tachibana S., Yagura S. (1995) G-related musculoskeletal spine symptoms in Japan Air Self Defense Force F-15 pilots. Aviat. Space Environ. Med., 66: 269-272.

16. Knudson R., McMillan D., Doucette D., Seidel M. (1998) A comparative study of G-induced neck injury in pilots of the F/A-18. A-7. and A-4. Aviat. Space Environ. Med., 59: 758-760. 
17. Kolstad F., Myhr G., Kvistad K.A., Nygaard O.P., Leivseth G. (2005) Degeneration and height of cervical discs classified from MRI compared with precise height measurements from radiographs. Eur. J. Radiol., 55: 415-420.

18. Malfair D., Beall D.P. (2007) Imaging the degenerative diseases of the lumbar spine. Magn. Reson. Imaging Clin. N. Am., 15: 221-238. vi.

19. Matsumoto M., Fujimura Y., Suzuki N., Nishi Y., Nakamura M. Yabe Y., Shiga H. (1998) MRI of cervical intervertebral discs in asymptomatic subjects. J. Bone Joint. Surg. Br., 80: 19-24.

20. Montgomery D.L. (2006) Physiological profile of professional hockey players - a longitudinal comparison. Appl. Physiol. Nutr. Metab., 31: 181-185

21.Newman D.G. (1997) +GZ-induced neck injuries in Royal Australian Air Force fighter pilots. Aviat. Space Environ. Med., 68: 520-524.

22. Oksa J., Rintamäki H., Mäkinen T., Hassi J., Rusko H. (1995) Cooling-induced changes in muscular performance and EMG activity of agonist and antagonist muscles. Aviat. Space Environ. Med., 66: 26-31.

23. Petren-Mallmin M., Linder J. (1999) MRI cervical spine findings in asymptomatic fighter pilots. Aviat. Space Environ. Med., 70: 1183-1188.

24. Petren-Mallmin M., Linder J. (2001) Cervical spine degeneration in fighter pilots and controls: a 5-yr follow-up study. Aviat. Space Environ. Med., 72: 443-446.

25. Rintala H., Häkkinen A., Siitonen S., Kyröläinen H. (2015) Relationships between physical fitness, flight duty and musculoskeletal symptoms in military pilots. Mil. Med., 180, 12: 1233-1238.
26. Santtila M., Kyröläinen H., Vasankari T., Tiainen S., Palvalin K., Häkkinen A., Häkkinen K. (2006) Physical fitness profiles in young Finnish men during the years 1975-2004. Med. Sci. Sports Exerc., 38: 1990-1994.

27. Sheard S.C., Pethybridge R.J., Wright J.M., McMillan G.H. (1996) Back pain in aircrew - an initial survey. Aviat. Space. Environ. Med., 67: 474-477.

28. Sovelius R., Salonen O., Lamminen A., Huhtala H., Hämäläinen O. (2008) Spinal MRI in Fighter Pilots and Controls: A 13-year Longitudinal Study. Aviat. Space Environ. Med., 79: 865-868.

29. Thalgott J.S., Albert T.J., Vaccaro A.R., Aprill C.N., Giuffre J.M., Drake J.S., Henke J.P. (2004) A new classification system for degenerative disc disease of the lumbar spine based on magnetic resonance imaging, provocative discography, plain radiographs and anatomic considerations. Spine J. 4: 167S-172S.

30. Videman T., Sarna S., Battie M.C., Koskinen S., Gill K., Paananen H., Gibbons L. (1995) The long-term effects of physical loading and exercise lifestyles on back-related symptoms, disability, and spinal pathology among men. Spine, 20: 699-709.

\section{Received 27.05.2017 \\ Accepted 11.09.2017}

(C) University of Physical Education, Warsaw, Poland 